\title{
Ganz andere Umstände: Schwangerschaft nach oder während einer Krebserkrankung
}

\author{
Krebs und Kinderwunsch schließen einander nicht mehr \\ von vornherein aus. Selbst wenn ein Tumor während der \\ Schwangerschaft diagnostiziert wird, muss diese heute nur \\ noch selten beendet werden. Voraussetzung ist jedoch eine \\ Behandlung in einem Krebszentrum, in dem interdisziplinäre \\ Behandlungskonzepte entwickelt werden können. Bei Patienten \\ mit Kinderwunsch sollte bei einer Tumorerkrankung rechtzeitig \\ an fertilitätssichernde Maßnahmen gedacht werden.
}

Nach Angaben des Deutschen Krebsforschungszentrums liegt das mittlere Erkrankungsalter für Krebs bei 69 Jahren für Männer und bei 68 Jahren für Frauen. Doch es gibt auch Tumorentitäten mit einem Erkrankungsgipfel im jüngeren oder mittleren Lebensalter wie beispielsweise Gebärmutterhals- oder Hodenkrebs. Bei Brustkrebs haben epidemiologische Studien gezeigt, dass zum Diagnose-Zeitpunkt $11 \%$ der Betroffenen zwischen 35 und 44 Jahre und $2 \%$ zwischen 20 und 34 Jahre alt sind. Parallel dazu gibt es unübersehbar den gesellschaftlichen Trend, dass Frauen, aber auch Männer, bei Geburt des ersten Kindes deutlich älter sind als vor Jahrzehnten. Ärzte werden daher immer häufiger mit dem Problem konfrontiert, dass die Familienplanung zum Zeitpunkt der Krebsdiagnose bzw. -behandlung noch nicht abgeschlossen ist.

Auswirkungen einer Krebsbehandlung auf die Familienplanung

Der Einfluss der Behandlungsstrategien bei Krebs auf die Fertilität ist sehr unterschiedlich und wird außerdem von individuellen Faktoren, vor allem dem Alter des Patienten, bestimmt. Auch die psychische Komponente darf nicht unterschätzt werden. So wünschen sich beispielsweise Menschen, die sich in ihrer Kindheit einer onkologischen Behandlung unterziehen mussten, seltener eigene Kinder. In einer Umfrage (Klin Pädiatr 2008; 220: 159-165) unter ehemaligen kinderonkologischen Patienten mit einem Durchschnittsalter von rund 24 Jahren lag die Kinderwunschrate bei 77 \% im Vergleich zu $90 \%$ in der altersentsprechenden Gesamtbevölkerung. Am häu-

figsten wurde von den potenziellen Eltern die Angst geäußert, dass das Kind auch an Krebs erkranken oder dass die eigene Krankheit neu ausbrechen könnte. Doch diese Ängste sind im Wesentlichen unbegründet, denn großangelegte Studien haben gezeigt, dass Nachkommen von ehemals krebserkrankten bzw. -therapierten Eltern (abgesehen von der genetischen Belastung bei Brustkrebs) kein höheres Risiko für Tumorerkrankungen besitzen. In der genannten Umfrage lagen die Fehlgeburtenrate, das Geburtsgewicht und der Kopfumfang bei Geburt im Normbereich. Von 30 Umfrageteilnehmern wurden 41 Kinder geboren bzw. gezeugt, von denen 40 gesund waren und nur eines eine Fußfehlstellung hatte.

Folgen von Krebsoperation, Bestrahlung und Chemotherapie Die Chancen für eine erfolgreiche Schwangerschaft nach einer Krebserkrankung haben sich dank moderner OP-Verfahren deutlich erhöht, erläutert Prof. Dr.

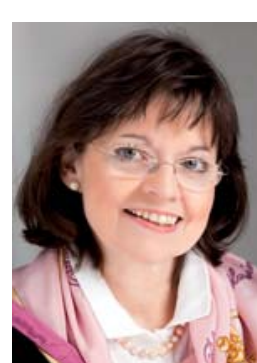

Prof. Dr. med. Eva-Maria Grischke med. Eva-Maria Grischke, Leiterin der Onkologie an der UniversitätsFrauenklinik Tübingen: „Früher wurde beispielsweise beim Ovarialkarzinom standardmäßig eine Ovarektomie und eine Hysterektomie durchgeführt. Heute ist es möglich, in frühen Stadien organerhaltend zu operieren, sodass ein Kinderwunsch realisiert werden kann. Nach der OP sollte die Patientin aber relativ schnell schwanger werden. Eventuell muss sich nach der Entbindung noch eine komplettierende Operation anschließen.“

Bei einer Bestrahlung im Unterleibsbereich hängt das Ausmaß einer möglichen Schädigung vom Alter der Patientin und der Strahlendosis ab. Die Wahrscheinlichkeit eines kompletten Verlustes der Ovarialfunktion steigt mit zunehmendem Alter. Frauen über 40 haben eine geringere Eizellreserve, bei ihnen muss bereits bei Strahlendosen zwischen 5 und 6 Gy mit einem dauerhaften Funktionsverlust gerechnet werden. Jüngere Frauen dagegen können unter Umständen bis zu 20 Gy tolerieren. Wird bei einem Mädchen oder einer Jugendlichen der Unterleib bestrahlt, kann es Untersuchungen zufolge zu einem reduzierten Uterusvolumen, einer geringeren Endometrium-Dicke oder einem Elastizitätsverlust der Uterusmuskulatur kommen. Bei Heranwachsenden kann eine Bestrahlung im Kopfbereich zu einer Störung der Hypothalamus-Hypophysen-Ovarien-Achse führen. Bei Männern kann es bereits bei Strahlendosen von 0,1 Gy zu einer temporären Oligospermie kommen. Höhere Dosen können zu einer kompletten Unterbrechung der Spermienproduktion führen, die - allerdings manchmal erst nach mehreren Jahren - reversibel ist. Strahlendosen über $6 \mathrm{~Gy}$ führen häufig zu einer dauerhaften Infertilität. Zur Höhe der schädigenden Strahlendosis finden sich in der Literatur jedoch sehr unterschiedliche Angaben.

Auch eine Chemotherapie kann gravierende Folgen haben. Wirkstoffe wie Busulfan, Carboplatin, Cisplatin, Cyclophosphamid, Ifosfamid, Etoposid, Melphalan und Procarbazin gelten als besonders fertilitätsschädigend. Auch wenn eine Chemotherapie für die Ovarien in der Regel

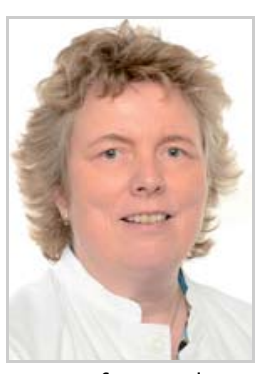

Univ.-Prof. Dr. med. Nadia Harbeck weniger toxisch ist als eine Radiotherapie, kann sie dennoch zu einer massiven Schädigung bis hin zur permanenten Amenorrhö führen. Alkylanzien wie beispielsweise Cyclophosphamid und Ifosfamid sind besonders gonadotoxisch, da sie unabhängig vom Zellzyklus wirken und daher auch ruhende Eizellen beeinträchtigen können. Univ.Prof. Dr. med. Nadia Harbeck, Leiterin des 
Brustzentrums der Universität München mit den Standorten Frauenkliniken Großhadern und Maistraße-Innenstadt, weiß aus ihrer Erfahrung, dass die Ovarien bei jungen Frauen eine Chemotherapie häufig problemlos überstehen und die Menstruation nach Abschluss der Behandlung meist wieder einsetzt.

Bei Männern ist die Widerstandsfähigkeit des Spermienepithels und der Testosteron-produzierenden Leydig-Zellen gegenüber einer Chemotherapie sehr unterschiedlich, das Ausmaß der Schädigung daher schwer vorhersagbar. Alkylanzien wie Cyclophosphamid oder Procarbazin sind Untersuchungen zufolge auch bei Männern gonadotoxisch. Auch bei Platin-haltigen Dosierungsschemata wurde eine verlängerte Azoospermie beobachtet. Therapien, die die genannten Substanzen nicht enthalten, verursachen wahrscheinlich keine dauerhafte Azoospermie.

Lange Wartezeiten nicht notwendig

„Bei Brustkrebspatientinnen hat man früher die Ansicht vertreten, dass nach einer Behandlung eine Wartezeit von zwei Jahren bis zur Schwangerschaft eingehalten werden muss“, erläutert Grischke. „Dieses Intervall gründete sich jedoch nur auf die statistische Wahrscheinlichkeit für das Rezidiv- und Metastasierungsrisiko, das in den ersten beiden Jahren nach der Behandlung am höchsten ist. Es gibt jedoch keine Daten, die das rechtfertigen. Daher wird heute die Ansicht vertreten, dass je nach individuellem Rezidiv- bzw. Metastasierungs-Risiko eine Wartezeit von sechs Monaten ausreichend sein kann."

Häufig wird vermutet, dass bei einer Schwangerschaft nach erfolgreich behandeltem Brustkrebs das Rezidivrisiko erhöht ist. Dazu Harbeck: „Das Mammakarzinom ist sicher eine der bestuntersuchten Erkrankungen, und es gibt gute Daten, dass sich das Rezidivrisiko durch die hormonellen Umstellungen in der Schwangerschaft nicht erhöht, das heißt eine Schwangerschaft nach Brustkrebs verschlechtert nicht die Heilungschancen. Es gibt aber auch Einschränkungen. Wird beispielsweise eine adjuvante Therapie wegen der Schwangerschaft früher abgesetzt, ist dies mit einer Einbuße der Heilungschancen vergesellschaftet. Eine solche Behandlung kann man aber nach der Schwangerschaft wieder aufnehmen und zu Ende führen. Man therapiert ja heute bei Brustkrebs bis zu zehn Jahre lang. Bei anderen Krebsarten kann sich das durchaus anders verhalten. Wenn es beispielsweise zu einer Metastasierung gekommen ist und man wichtige Therapien unterbrechen müsste, würde man eher von einer Schwangerschaft abraten, weil man nicht neun Monate Zeit hätte, um eine überlebenssichernde Therapie aufzuschieben. Eine erwähnenswerte Besonderheit ist der so genannte HealthyMother-Effekt, was bedeutet: Die Wahrscheinlichkeit für eine Schwangerschaft ist bei solchen Patientinnen höher, die per se eine bessere Heilungschance haben."

\section{Fertilitätserhaltende Maßnahmen} vor der Krebstherapie

Vor einer Krebstherapie sollte bei Patienten mit Kinderwunsch über Verfahren gesprochen werden, die die Fertilität nach Abschluss der Behandlung gewährleisten oder verbessern. Bei männlichen Krebspatienten sind diese fertilitätserhaltenden Maßnahmen deutlich unkomplizierter als bei Patientinnen, da die Kryokonservierung von Spermien einfach und vergleichsweise kostengünstig durchführbar ist. Mit den Methoden der assistierten Reproduktion (In-vitro-Fertilisation, IVF, oder Intrazytoplasmische Spermieninjektion, ICSI) können die Keimzellen bei Bedarf eingesetzt werden. Hat eine Tumorbehandlung $\mathrm{zu}$ einer Azoospermie geführt, können Spermien auch mithilfe der testikulären SpermienExtraktion (TESE) operativ aus dem Hodengewebe gewonnen werden.

Auch für Frauen steht heute eine Reihe von fertilitätserhaltenden Verfahren zur Verfügung:

- Eierstockschutz mittels GnRH-Agonisten

- hormonelle Stimulation mit anschließender Kryokonservierung der Eizellen

- laparoskopische Entnahme von Eierstockgewebe mit dem Ziel der späteren Re-Transplantation

- Oophoropexie (Transposition der Ovarien): chirurgische Technik, bei der die Eierstöcke vor einer geplanten Beckenbestrahlung aus dem Strahlungsfeld entfernt werden.

Bei befruchteten kryokonservierten Eizellen ist die Chance auf eine Schwangerschaft altersabhängig. Sie wird bei Patientinnen zwischen 18 und 25 Jahren mit $40 \%$ angegeben und sinkt mit steigen- dem Alter auf ca. $25 \%$ bei Frauen zwischen 36 und 40 (Schweiz Med Forum 2012; 12: 708-709). Um die derzeit verfügbaren Möglichkeiten der Fertilitätsprotektion $\mathrm{zu}$ bündeln, $\mathrm{zu}$ optimieren und wissenschaftlich $\mathrm{zu}$ evaluieren, hat sich 2006 das Netzwerk FertiPROTEKT gegründet. Derzeit sind darin 100 IVFZentren oder -Kliniken, d.h. fast $70 \%$ der deutschen IVF-Zentren, aktiv (www.fertiprotekt.de).

\section{Krebstherapie während der \\ Schwangerschaft}

„Werden Patientinnen während der Schwangerschaft mit einer Krebserkrankung konfrontiert, gehören sie in ein großes Zentrum, in dem alle Expertisen einschließlich Pränataldiagnostik, Onkologie und Psychoonkologie vor Ort sind“, betont Harbeck. „Wenn eine Patientin jung ist und einen Kinderwunsch hat, muss bereits beim Erstgespräch an die fertilitätssichernden Maßnahmen gedacht werden. Denn dafür ist ein Vorlauf von zwei bis drei Wochen notwendig eine Zeitspanne, die wir beim Brustkrebs zur Verfügung haben, bei anderen Erkrankungen wie beispielsweise hämatologischen Tumoren jedoch nicht." Eine Brustkrebserkrankung könne heute in der Schwangerschaft fast genauso behandelt werden wie außerhalb, die Heilungschancen für die Patientin sind identisch. „Bei der Chemotherapie gibt es gute Daten für Anthrazykline, sowie - wenn auch in geringerem Umfang - für Taxane. Hier wissen wir, dass die Kinder gesund zur Welt kommen. Es ist bei Brustkrebs auch nicht notwendig, das Kind vorzeitig zu holen. Notwendige Krebsdiagnostik wie bildgebende Verfahren können dann nach der Entbindung nachgeholt werden."

Dr. rer. nat. Claudia Bruhn, Schmölln

DOI 10.1055/s-0034-1374689

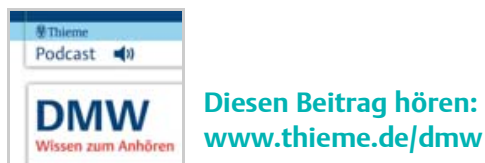

Dtsch Med Wochenschr 2014; 139, Nr. 22 